\title{
MicroRNA-487b-3p inhibits osteosarcoma chemoresistance and metastasis by targeting ALDH1A3
}

\author{
MING CHENG, PING-GUO DUAN, ZHI-ZENG GAO and MIN DAI \\ Department of Orthopedics, The First Affiliated Hospital of Nanchang University, Nanchang, Jiangxi 330006, P.R. China
}

Received July 10, 2019; Accepted February 21, 2020

DOI: $10.3892 /$ or.2020.7814

\begin{abstract}
Metastasis and chemoresistance indicate poor prognosis in patients with osteosarcoma (OS). In the present study, the expression level of microRNA(miR)-487b-3p in OS specimens and cell lines was found to be decreased, and the expression level of miR-487b-3p was associated with overall survival in patients with OS. The inhibition of miR-487b-3p stimulated OS cell migration and contributed to the development of chemoresistance. In contrast, the overexpression of miR-487b-3p significantly inhibited OS cell migration and enhanced the sensitivity of OS cells to doxorubicin treatment. In addition, the results from the present study revealed that the suppression of miR-487b-3p stimulates OS stemness, while the overexpression of miR-487b-3p suppresses OS stemness. Notably, in vivo experiments also revealed that the overexpression of miR-487b-3p inhibited cancer stem cell (CSC)-induced tumor formation, and the combination treatment of miR-487b-3p and doxorubicin significantly inhibited CSC-induced tumor growth. Furthermore, miR-487b-3p exerts its anticancer role by targeting aldehyde dehydrogenase 1 family member A3 in OS. Taken together, the results from the present study suggests that miR-487b-3p is a tumor suppressor and that the overexpression of $\mathrm{miR}-487 \mathrm{~b}-3 \mathrm{p}$ is a novel strategy to inhibit tumor metastasis and chemoresistance in OS.
\end{abstract}

\section{Introduction}

Osteosarcoma (OS) is the most common primary malignant tumor, with high incidence rates in children and adolescents for nearly 20 years, in the world (1). Cisplatin, doxorubicin (Dox) and methotrexate (MTX) are commonly used anticancer drugs for OS in a clinical setting (2), and patients who

Correspondence to: Professor Zhi-Zeng Gao or Professor Min Dai, Department of Orthopedics, The First Affiliated Hospital of Nanchang University, 17 Yongwaizheng Street, Nanchang, Jiangxi 330006, P.R. China

E-mail: 570480692@qq.com

E-mail: daimim@medmail.com.cn

Key words: microRNA-487b-3p, ALDH1A3, chemoresistance, metastasis, osteosarcoma do not respond to these drugs have a poor prognosis (3). Lung metastasis is present in $20 \%$ of patients with OS at time of diagnosis, and current active treatments, including chemoand radio-therapy, do not guarantee long-term survival, for example $>5$ years $(4,5)$, suggesting that chemoresistance and metastasis are still the primary challenges in the treatment of OS. However, the mechanisms leading to chemoresistance and metastasis development in OS remain unclear.

Cancer stem cells (CSCs) are a small subset of cells located within a tumor, and studies show that CSCs are implicated in chemotherapy resistance and metastasis in cancer such as non-small cell lung cancer and breast cancer (6-8). CSCs present high resistance to chemotherapy drugs that are commonly used in the treatment of OS, including cisplatin and doxorubicin (5,7). In addition, studies have revealed that CSCs are able to regenerate all of the cell types (HuO9, HuO9-M132, Saos-2, Saos-2-LM5 and MCF7, SKBR3, MDA-MB-231, MDA-MB-435) in the tumor due to their stem cell-like behavior, resulting in metastatic relapse $(5,8)$. Therefore, CSCs are important therapeutic targets in cancer. However, the mechanism of CSC regulation in OS remains unknown.

MicroRNAs (miRNA/miR) are a class of non-coding RNAs that are 18-22 nucleotides in length and negatively regulate gene expression through the interaction with the 3' untranslated region (UTR) of the target gene mRNA (9). Dysregulated expression of miRNAs (miR-125b, miR-199a, let-7 g, miR-433, miR-214 and miR-100) has been detected in most types of cancer, and dysregulation of even a single miRNA can lead to tumorigenesis and stimulate cancer progression $(10,11)$. In addition, dysregulated expression of miRNAs (let-7, miR-135 and miR-200) was demonstrated in CSCs, and such aberrantly regulated miRNAs are involved in the development of CSCs and the maintenance of stemness $(6,12)$. Decreased expression levels of miR-382, miR-369, miR-134, miR-127-3p, located on chromosome 14q32, have been identified in OS (13), and previous studies have revealed that the expression level of these miRNAs is associated with the progression of OS, including rapid growth of the tumor, recrudescence and metastasis $(13,14)$. miR-487b-3p is one of the aforementioned miRNAs and is associated with progression of OS; however, its function and mechanism in OS remains unknown. In the present study the functional role of miR-487b-3p was determined and the miRNA was found to be a tumor inhibitor, regulating invasion, chemoresistance and the development of CSCs by targeting ALDH1A3 in 
OS. ALDH1A3 plays an important role in cancer stemness maintenance and has been associated with the development, progression and prognosis of different cancer types (15-17). In addition, ALDH1 A3 is involved with a diverse range of biological characteristics within cancer stem cells and can act as a marker for these cells (17). ALDH1A3 could therefore provide novel targets for the treatment of osteosarcoma.

\section{Materials and methods}

Cell culture and human specimens. All osteosarcoma cells (U2OS, Saos2, MG63, HOS) and the 293T cell line were purchased from the American Type Culture Collection and cultured in RPMI 1640 media (Sigma-Aldrich; Merck KGaA) supplemented with $10 \%$ fetal bovine serum (HyClone; GE Healthcare). Human specimens were collected during diagnostic biopsies (paraffin embedded tissue) at First Affiliated Hospital of Nanchang University (Jiangxi, China) between May 2017 and September 2018. A total of 40 diagnostic patient specimens and 24 healthy bone specimens were used in the present study (Table I), which were also obtained from First Affiliated Hospital of Nanchang University (Jiangxi, China). All the OS cases were diagnosed by pathological evaluation using the National Comprehensive Cancer Network Guidelines version 2.2017 (18) and had complete clinical information. The TNM stage was graded according to the eighth edition of American Joint Committee on Cancer TNM staging (19). Written informed consent was provided by all the participants. Ethics approval was granted by the Research Ethics Board of the First Affiliated Hospital of Nanchang University (approval no. 2019042).

Transfection. Cell transfection was performed using Lipofectamine ${ }^{\circledR} 3000$ transfection reagent according to manufacturer's protocol (Invitrogen; Thermo Fisher Scientific, Inc.) and the protein or total RNA was extracted after $72 \mathrm{~h}$. miR-487b-3p mimics (5'-AAUCGUACAGGGUCAUCCACU U-3'), inhibitors (5'-AAGUGGAUGACCCUGUACGAUU-3') and negative control (NC) oligonucleotides (5'-UUCUCC GACGUGUCACGUTT-3'), the aldehyde dehydrogenase 1 family member A3 (ALDH1A3) expression vector (pcDNA3.1 plasmid), and the ALDH1A3 short inhibiting (si)RNA (5'-GCA GAGAACUAGGUGAAUAdTdT-3') and control siRNA (5'-GTATCGAAGAAGTGATAAA-3') were purchased from Guangzhou RiboBio Co., Ltd.. The concentration of miRNA and siRNA used was $50 \mathrm{pM} / 96$-well, and for the plasmid was $5 \mu \mathrm{g} / 6$-well.

For transfection of Saos 2 cells, they were seeded into $60 \mathrm{~mm}$ dishes and incubated overnight at $37^{\circ} \mathrm{C}$ in humidified incubator with $5 \% \mathrm{CO}_{2}$. Following which, $10 \mu \mathrm{l}$ lentivirus with a titer of $1 \times 10^{8} \mathrm{TU}$ was added and the cells were incubated at $37^{\circ} \mathrm{C}$ in a humidified incubator with $5 \% \mathrm{CO}_{2}$ for $72 \mathrm{~h}$. Lastly, puromycin, at a final concentration of $2 \mu \mathrm{g} / \mathrm{ml}$ was added and the cells were cultured for $48 \mathrm{~h}$ to select the transfected cells.

$R N A$ extraction and reverse transcription-quantitative $P C R$ $(R T-q P C R)$. Total RNA was extracted from cells or tissues using TRIzol ${ }^{\circledR}$ reagent (Thermo Fisher Scientific, Inc). miR-487b-3p and RNU6 expression was detected using RT-qPCR using SYBR-Green and the following primers purchased from
Guangzhou RiboBio Co., Ltd: miR-487b-3p forward, 5'-AATCGTACAGGGTCATC-3 and reverse, 5'-GTGCAG GGTCCGAGGT-3'; U6 forward, 5'-GCTTCGGCAGCACAT ATACTAAAAT-3' and reverse 5'-CGCTTCACGAATTTG CGTGTCAT-3'. The sequences of the primers for ALDH1A3 and GAPDH are as follows: ALDH1A3 forward, 5'-TGAGTG ATTTAGCAGGCTGCA-3', and reverse 5'-TGGCCACAT ACACCAATAGGTTC-3'; and GAPDH forward 5'-GCAGGG GGGAGCCAAAAGGGT-3' and reverse 5'-TGGGTGGCA GTGATGGCATGG-3'. miRNA was reverse transcribed using the polyA tailing method (All-in-One ${ }^{\mathrm{TM}}$ miRNA RT-qPCR detection kit), while mRNA was reverse transcribed using the All-in-One ${ }^{\mathrm{TM}}$ first-strand cDNA synthesis kit, (both from GeneCopoeia, Inc.). D The following temperature protocol was used: Incubation at $37^{\circ} \mathrm{C}$ for $60 \mathrm{~min}$, and $72^{\circ} \mathrm{C}$ for $5 \mathrm{~min}$, then the samples were store at $4^{\circ} \mathrm{C}$. The following thermocycling conditions were used: Initial denaturation at $95^{\circ} \mathrm{C}$ for $10 \mathrm{~min}$, followed by 40 cycles of $95^{\circ} \mathrm{C}$ for $10 \mathrm{sec} .58^{\circ} \mathrm{C}$ for $20 \mathrm{sec}$ and $72^{\circ} \mathrm{C}$ for $15 \mathrm{sec}$. The relative expression levels of miR487b-3pa and ALDH1A3 were normalized against RNU6 and GAPDH, respectively. The $2^{-\Delta \Delta \mathrm{Cq}}$ method was used to analyze the relative fold-changes (20).

Apoptosis analysis. Saos-2 cells were seeded in 6-well plates, at a density of $2 \times 10^{5}$, after $6 \mathrm{~h}$ of transfection. Cells were incubated with or without Dox $(0.2 \mathrm{uM})$. The cells were harvested and stained with annexin $\mathrm{V}$ and 7-aminoactinomycin D (7-AAD) at room temperature for $30 \mathrm{~min}$, according to the manufacturer's protocol in (Biotium Inc.) $24 \mathrm{~h}$ later. The cells were subsequently analyzed using flow cytometry (CytoFLEX) and analyzed using CytoFLEX v2.0 software (both Beckman Coulter, Inc).

MTT assay. The MTT assay was performed according to the manufacturer's protocol using MTT assay kit (Promega Corporation). SAOS2 cells were transfected with miR-487b-3p mimics, inhibitors and NC oligonucleotides using Lipofectamine ${ }^{\circledR} 3000$ (Invitrogen; Thermo Fisher Scientific, Inc.) according to manufacturer's instructions. After $6 \mathrm{~h}$ of transfection, cells were reseeded in a 96-well plate at a density $10,000 /$ well. After $12 \mathrm{~h}$ of incubation, cells were treated with $0.2 \mu \mathrm{M}$ doxorubicin for $24 \mathrm{~h}$, after which the medium was removed and $100 \mu \mathrm{l}$ DMSO was added. The absorbance was measured at $570 \mathrm{~nm}$.

Transwell and osteosphere assays. The Transwell and osteosphere assays were performed as described previously by $\mathrm{Xu}$ et al (5). Briefly, $1 \times 10^{5}$ transfected cells in serum-free growth DMEM were seeded in the upper wells of the chamber (12-well plate). The lower wells contained the same medium with $10 \%$ serum. After $24 \mathrm{~h}$, the cells that had migrated to the lower side of the chamber were fixed with $2.5 \%$ glutaraldehyde for $15 \mathrm{~min}$, stained with $0.1 \%$ crystal violet for $30 \mathrm{~min}$ at room temperature and counted using ImageJ software (v1.8.0; National Institutes of Health), from 3 fields of view. For the osteosphere assay, 1,000 cells were plated in 24-well ultra-low attachment plates in N2B27-defined serum free medium and cultured for 9 days at $37^{\circ} \mathrm{C}$ in a humidified incubator with $5 \% \mathrm{CO}_{2}$. Spheres were counted in each plate using a Leica MZ12 inverted light microscope, at $\mathrm{x} 4$ magnification, and using 3 fields of view. 
Table I. Characteristics of healthy control and patients with OS.

\begin{tabular}{lcc}
\hline Variable & Patients & Healthy controls \\
\hline Sex, $\mathrm{n}$ & & 13 \\
Male & 26 & 11 \\
Female & 14 & 0.365 \\
Mean age \pm SD, years (range) & $40.2 \pm 4.5(22-67)$ & $42.3 \pm 3.8(21-62)$ \\
Age, years, $\mathrm{n}$ & & 10 \\
$\leq 35$ & 22 & 14 \\
$>35$ & 18 & - \\
TNM stages, $\mathrm{n}$ & & - \\
I-II & 24 & 0.314 \\
III-IV & 16 & - \\
Histology, $\mathrm{n}^{\mathrm{a}}$ & & - \\
Poor & 9 & - \\
Moderate & 17 & 24 \\
Well & 14 & \\
Total & 40 & \\
\hline
\end{tabular}

$\mathrm{a}(15)$

Luciferase reporter assay. To identify target genes of miR-487b-3p, the online database miRDB (www.mirdb.org) was used. A total of 3'UTRs from ALDH1A3 were predicted to interact with miR-487b-3p and these were amplified using PCR from human genomic DNA (purchased from Shanghai GenChem Co., Ltd.) and ligated into the Luciferase reporter vector (pGL4; Hanheng Biotechnology Co., Ltd) using the HindIII and SacI restriction sites. The primer sequences for amplifying the 3'UTR of ALDH1A3 are as follows: forward, 5'-AAAGATCCTTTATTAAGCTTTAATAAAATG AGGGCCCGTAACAGAACCAGTG-3'; and reverse, 5'-GCG CACTAGTGAGGGAGCTCTTGTGGGATGCGATCTGCA GCTAGGA-3'.

For the luciferase reporter experiments, the 293T cells were seeded into 24 -well cell culture plates and transfected with the aforementioned luciferase reporter plasmids and either the miR-487b-3p or NC oligonucleotides, using Lipofectamine ${ }^{\circledR} 3000$ (Invitrogen; Thermo Fisher Scientific, Inc.). Cells were subjected to a luciferase reporter assay, $48 \mathrm{~h}$ following incubation. Luciferase activity was measured using the dual-luciferase assay system (Promega Corporation) according to the manufacturer's protocol. The luminous intensity of firefly fluorescein was normalized with the luminous intensity of Renilla fluorescein as a reference, then the data was analyzed. Renilla luciferase was used as an internal reference. The relative value was used as the control.

Western blot analysis. The cells were lysed using RIPA (Sigma-Aldrich; Merck KGaA), and subsequently quantified using bicinchoninic acid (Beijing Dingguo Changsheng Biotechnology Co. Ltd). A total of $25 \mu \mathrm{g}$ of protein was separated using 10\% SDS-PAGE, and the proteins were transferred to PVDF membrane, which were subsequently blocked with $5 \%$ skimmed milk for $1 \mathrm{~h}$ at room temperature.
The primary antibodies E-cadherin (cat. no. 17952-1-AP; 1:1,000, ProteinTech Group, Inc.), vimentin (cat. no. 5471; 1:2,000; Cell Signaling Technology, Inc.), cleaved-PARP (cat. no. 5625; 1:1,500; Cell Signaling Technology, Inc.), PARP (cat. no. ab74290; 1:3,000; Abcam), ALDH1A3 (cat. no. ab129815; 1:800; Abcam), SOX-2 (cat. no. 11064-1-AP; 1:2,000; ProteinTech Group, Inc.), OCT-4 (cat. no. 11263-1-AP; 1:2,000; ProteinTech Group, Inc.), polycomb complex protein BMI-1 (BMI-1; cat. no. ab38295; 1:2,000 Abcam), $\beta$-actin (cat. no. 3700; 1:5,000; Cell Signaling Technology, Inc.), were used and incubated overnight at $4^{\circ} \mathrm{C}$. Following which, the membranes were incubated with secondary antibodies (goat anti-rabbit; cat. no, A16104; 1:10,000; goat anti-mouse; cat. no, 31430; 1:10,000) (both Thermo Fisher Scientific, Inc.) conjugated to horseradish peroxidase for $2 \mathrm{~h}$ at room temperature. Then the proteins were visualized using an ECL kit (Bio-Rad Laboratories, Inc.) and quantified using Quantity One software (v4.6.2; Bio-Rad Laboratories, Inc.)

CD133 detection. The transplanted tumor tissue was washed with Hanks' buffer then cut into $3 \mathrm{~mm}^{3}$ sections. A total of $3 \mathrm{ml}$ serum-free RPMI-1640 medium (Sigma-Aldrich; Merck $\mathrm{KGaA}$ ) was subsequently added followed by $3 \mathrm{ml}$ collagenase $(0.1 \%)$ and the tissue sections were incubated for $3 \mathrm{~h}$ at $37^{\circ} \mathrm{C}$ for digestion. Following which, the tissue fragments were passed through a $70-\mu \mathrm{m}$ cell sieve to produce a cell suspension, for cell testing. The cells were resuspended with RPMI 1640 medium and centrifuged for $10 \mathrm{~min}$ at $200 \mathrm{x} \mathrm{g}$ at $4^{\circ} \mathrm{C}$ and washed once with PBS. The four groups of cells obtained were adjusted to a concentration of $1 \times 10^{6}$ per $100 \mu 1$ and transferred to Eppendorf ${ }^{\circledR}$ tubes $5 \mathrm{ml}$, following which, $1 \mu \mathrm{l}$ fragment crystallizable block antibody (BD Biosciences) was added and the tubes were incubated for $30 \mathrm{~min}$ at $4^{\circ} \mathrm{C}$ then washed once with PBS. A total of $5 \mu \mathrm{g}$ CD133 antibody (cat. no. ab252126; 
1:2,000; Abcam) was added to each tube and incubated for $30 \mathrm{~min}$ in the dark. Subsequently, $2 \mathrm{ml}$ cell staining buffer was added, and the samples were centrifuged at $350 \mathrm{x} \mathrm{g}$ for $5 \mathrm{~min}$ at $4^{\circ} \mathrm{C}$, twice. The cells were resuspended in $0.4 \mathrm{ml}$ cell staining buffer, following which $2 \mu 1$ nuclear dye 7-AAD (BD Biosciences) was added, and the cells were incubated on ice for 3-5 min, then analyzed using flow cytometry.

Animal experiments. ALDH $1^{\text {high }}$ cells were sorted from Saos-2 cells using FACS and the ALDEFLUOR kit (Stemcell Technologies, Inc.) as aforementioned. All animal experiments were performed using 6-week-old female athymic (nu/nu) mice $(17 \pm 1.5 \mathrm{~g})$ and housed under specific pathogen-free conditions, with $12 \mathrm{~h}$ light/dark cycles, $24^{\circ} \mathrm{C}$, $70 \%$ humidity and ad libitum access to food and water, and the veterinarian monitored the health and behavior of the animals every morning. For tumor formation experiments, $1 \times 10^{4}$ ALDH $1^{\text {high }}$ Saos- 2 cells that were previously transfected with miR-487b-3p mimics or NC in $100 \mu \mathrm{l}$ serum-free DMEM were subcutaneously (s.c.) injected into the back of the mouse, on the right side. After 2 months the mice were sacrificed using cervical dislocation. For chemotherapy experiments, $1 \times 10^{4}$ ALDH $1^{\text {high }}$ Saos- 2 cells in $100 \mu \mathrm{l}$ serum-free medium were s.c. injected into the back of the mouse, on the right side. When the tumors reached $\sim 50 \mathrm{~mm}^{3}$ (using Vernier caliper and measured every other day; volume $=$ long diameter $\mathrm{x}$ short diameter $\left.^{2}\right)$, mice were intraperitoneally injected with Dox (6 mg/ $\mathrm{kg}$ body weight), $10 \mu \mathrm{M}$ miR-487b-3p mimic or both, for three cycles (days 29, 32 and 36) and each group included nine mice (total 36 mice). Euthanasia was performed in mice when the tumor size reached $1,500 \mathrm{~mm}^{3}$. All animal experiments were approved by the Laboratory Animal Welfare and Ethics Committee of the Nanchang University (approval no. AMUWEC2018410).

Statistical analysis. Kaplan-Meier survival analysis was used to analyze the overall survival rate in patients with OS. Significant differences between treatment groups were analyzed using Student's t-test or ANOVA, followed by Tukey's post hoc test. All data were analyzed using the SAS statistical software package version 6.12 (SAS Institute, Inc.). $\mathrm{P}<0.05$ was considered to indicate a statistically significant difference.

\section{Results}

Decreased expression of miR-487b-3p is associated with poor clinical outcome. Previous studies have shown that $\sim 9$ miRNAs at the 14q32 locus are inversely correlated with OS progression (metastasis and relapse) $(13,14)$, which suggests that miR-487b-3p may also be associated with progression of OS. As expected, miR-487b-3p expression was significantly decreased in OS specimens compared with that in normal bone tissues (Fig. 1A). In addition, the clinical data also found that decreased expression of miR-487b-3p was significantly associated with poor overall survival in patients with $\mathrm{OS}(\mathrm{P}=0.0236)$ (Fig. 1B). Consistent with these results, miR-487b-3p expression was significantly decreased in OS cell lines compared with that in the human fetal osteoblastic hFOB1.19 cell line (Fig. 1C). Taken together, these data suggest that miR-487b-3p may play a role in OS, as a tumor suppressor.
miR-487b-3p inhibits invasion and chemoresistance in $O S$. The occurrence of metastasis and chemoresistance indicates a poor clinical outcome in patients with OS $(3,5)$. Thus, the effects of miR-487b-3p on OS metastasis and chemoresistance using OS cell lines transfected with miR-487b-3p mimic or inhibitor was investigated (Fig. S1 and Fig. 2). Transwell experiments demonstrated that the overexpression of miR-487b-3p significantly suppressed cell migration while the inhibition of miR-487b-3p stimulated cell invasion in both Saos-2 (Fig. 2B) and MG63 cells (Fig. S1B). In addition, the effects of miR-487b-3p on OS cell epithelial-mesenchymal transition (EMT) was also investigated, as EMT plays an important role in cancer metastasis. miR-487b-3p mimic was found to significantly increase E-cadherin expression in Saos-2 and MG-63 cells but significantly decreased vimentin expression (Fig. 2C and Fig. S1C, respectively), suggesting that miR-487-3p inhibits EMT in OS cells. Subsequently, the effects of miR-487b-3p on the chemotherapy sensitivity of OS cells was investigated. The results revealed that the increased level of miR-487b-3p significantly enhanced the sensitivity of Saos-2 and MG63 cells to Dox treatment compared with that the control cells, while the inhibition of miR-487b-3p inhibited Dox-induced cell viability inhibition (Fig. 2D and Fig. S1D). In addition, the results from the apoptosis analysis revealed that increased expression level of miR-487b-3p increased the rate of apoptosis in OS cells and the combination of miR-487b-3p and Dox significantly increased the rate of apoptosis compared with that in a single treatment (Fig. 2E and Fig. S1E). In contrast, the inhibition of miR-487b-3p significantly attenuated Dox-induced apoptosis in OS cells (Fig. 2E and Fig. S1E). Consistent with apoptosis analysis, combined treatment of miR-487b-3p and Dox significantly increased the protein expression level of the pro-apoptotic cleaved PARP protein compared with that in the Dox and mimic single treatment groups, while the miR-487b-3p inhibitor and Dox treatment groups significantly decreased the protein level of cleaved PARP compared with that in the Dox only treatment group in both Saos-2 and MG63 cells (Fig. 2F and Fig. S1F). Taken together, the results from the present study suggest that miR-487b-3p inhibits OS progression by inhibiting OS cell migration and inducing chemosensitivity.

miR-487b-3p inhibits CSCs in OS. Previous studies have suggested that CSCs lead to the development of metastasis and chemoresistance in multiple cancers, such as breast cancer and NSCLC $(7,10)$, thus, it was investigated whether miR-487b-3p is involved in CSC regulation of OS. As expected, the osteosphere assay revealed that the inhibition of miR-487b-3p significantly increased, while the overexpression of $\mathrm{miR}-487 \mathrm{~b}-3 \mathrm{p}$ reduced osteosphere numbers in Saos-2 (Fig. 3A) and MG63 cells (Fig. S2A). Consistent with sphere formation, the western blot results also showed that the overexpression of $\mathrm{miR}-487 \mathrm{~b}-3 \mathrm{p}$ significantly inhibited the expression level of the CSC marker proteins, including ALDH1, SOX2, OCT4 and BMI-1 (Figs. 3B and S2B). In contrast, the inhibition of miR-487b-3p significantly increased protein expression levels of the aforementioned CSCs marker (Figs. 3B and S2B). These data suggested that miR-487b-3p exerts its anticancer effects partially due to the inhibition of CSCs in OS. 
A

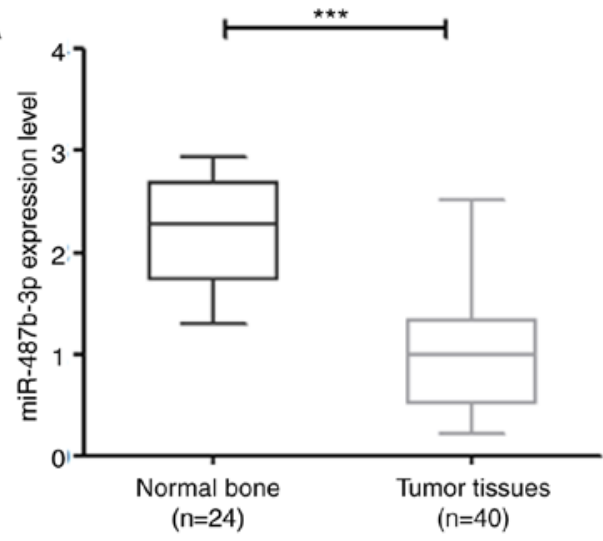

B

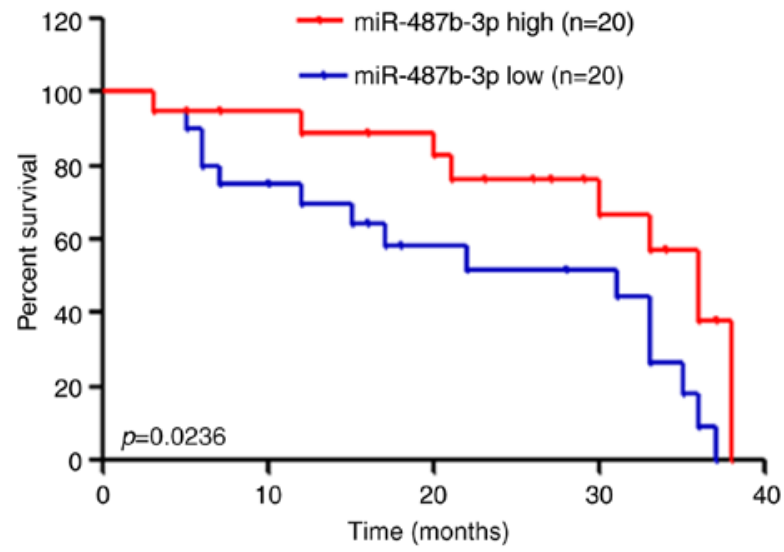

C

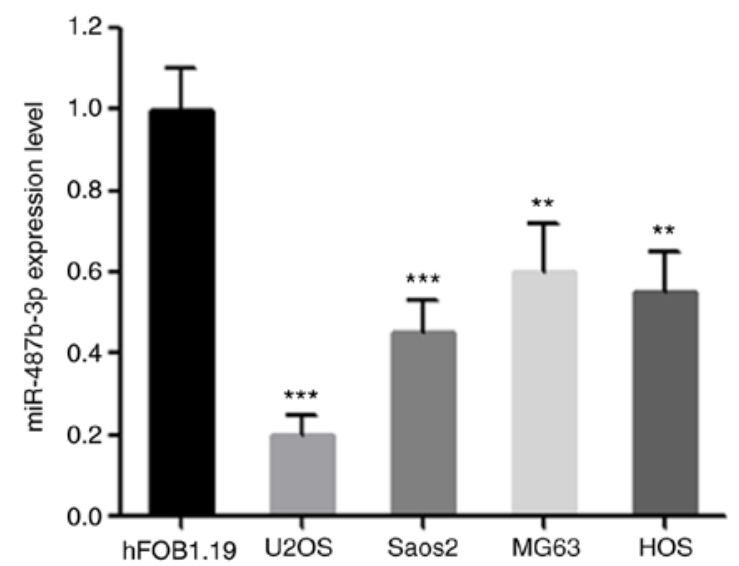

Figure 1. Decreased expression of miR-487b-3p is associated with poor clinical outcomes in patients with OS. (A) miR-487b-3p expression level was significantly downregulated in OS tumor tissues. (B) Kaplan-Meier survival rates in patients with OS with low $(\mathrm{n}=20)$ and high $(\mathrm{n}=20)$ miR-487b-3p expression level. (C) OS cell lines showed a low expression level of miR-487b-3p compared with that in human fetal hFOB1.19 osteoblastic cells. ${ }^{* *} \mathrm{P}<0.01$; ${ }^{* * *} \mathrm{P}<0.001$. miR, micro RNA; OS, osteosarcoma.

miR-487b-3p can target ALDH1A3. To investigate how miR-487b-3p regulates CSCs in OS, target gene candidates of $\mathrm{miR}-487 \mathrm{~b}-3 \mathrm{p}$ were investigated and multiple targets were identified, including ALDH1A3 (Fig. 4A). Thus, ALDH1A3 was chosen for further investigation. To investigate whether miR-487b-3p is involved in the regulation of ALDH1A3, ALDH1A3 protein and mRNA expression levels were determined after OS cells were transfected with miR-487b-3p mimic or inhibitor. The results showed that ALDH1A3 expression was significantly upregulated by the inhibition and downregulated by the overexpression of miR-487b-3p, in OS cells at both the mRNA and protein levels (Fig. 4B and C). Consistent with the in vitro results, the clinical sample analysis results also showed an inverse association between ALDH1A3 and miR-478b-3p expression levels in OS specimens $\left(\mathrm{R}^{2}=0.4471 ; \mathrm{P}=0.0129\right.$; Fig. 4D). Furthermore, it was verified that miR-487b-3p can directly target the 3'UTR of ALDH1A3 using the luciferase reporter assay, as the expression of ALDH1A3 was decreased in the WT group compared with that in the control group, indicating that miR-487b-3p can directly bind to the 3'UTR of ALDH1A3 to regulate its expression (Fig. 4E). These findings indicate that miR-487b-3p inhibits the mRNA and protein expression levels of ALDH1A3 by binding to its 3'UTR.

ALDHIA3 is a functional target of miR-487b-3p which modulates OS stemness. It was subsequently investigated whether ALDH1A3 is involved in the miR-487b-3p-mediated regulation of CSCs in OS. The expression of ALDH1A3 in OS cells was disrupted by transfection of ALDH1A3 expression vector or siRNA against ALDH1A3 (Fig. S3A). The overexpression or silencing of ALDH1A3 did not affect the miR-487b-3p expression (Fig. S3B and S3C). The overexpression of ALDH1A3 restored the downregulated expression of CSC marker proteins induced by miR-487b-3p, including ALDH1, SOX-2, OCT-4 and BMI-1 (Fig. 5A). In contrast, silencing of ALDH1A3 suppressed the protein expression levels of CSC markers, which were increased following inhibition of miR-487-3p (Fig. 5B). Consistent with these results, the overexpression of ALDH1A3 attenuated the miR-487b-3p-induced antisphere formation effect, while silencing of ALDH1A3 attenuated miR-487b-3p inhibition-induced stimulation of osteosphere formation (Fig. 5C). Together, the results have shown that miR-487b-3p regulates CSCs due to regulation of ALDH1A3 in OS.

miR-487b-3p significantly inhibits CSCs-induced tumorigenesis and chemoresistance in vivo. The in vitro experimental results indicated that upregulation of miR-487b-3p significantly inhibits OS stemness. Thus, the effects of miR-487b-3p on CSC-induced tumorigenesis and chemoresistance in vivo were investigated. ALDH $1^{\text {high }}$-expression cells were separated from OS cell lines using flow cytometry and transfected 
A

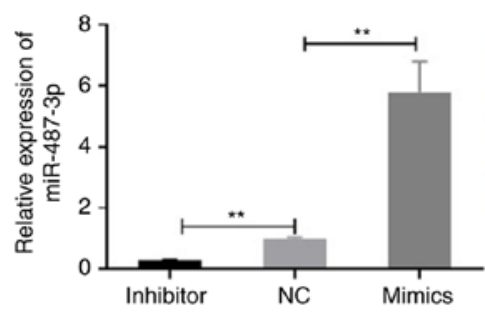

C

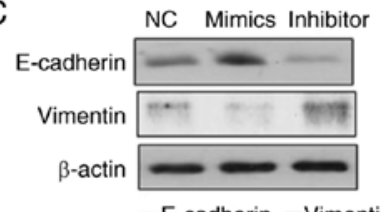

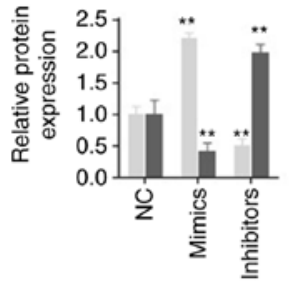

B

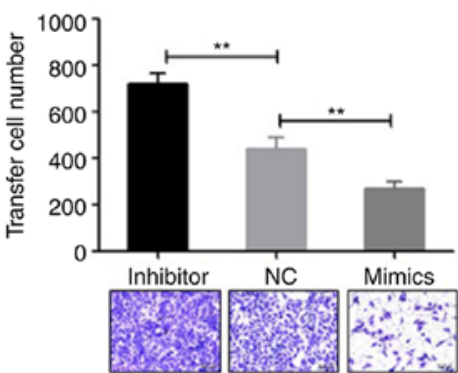

$\mathrm{D}$

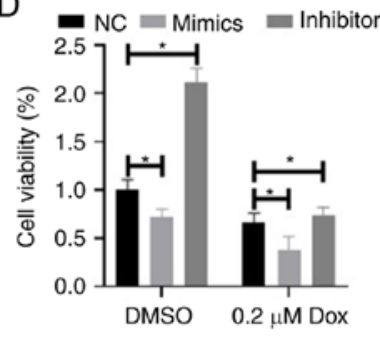

E
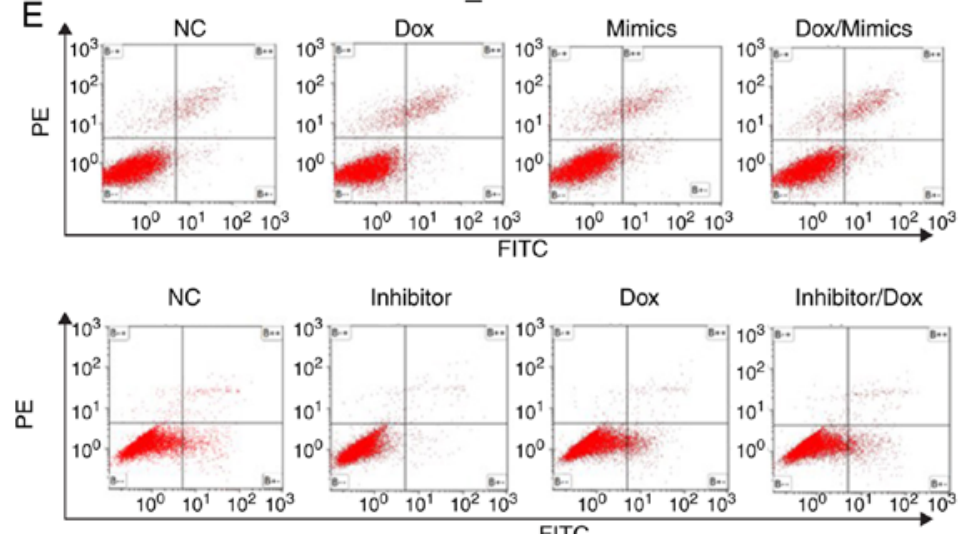

Dox

Dox Inhibitor/Dox

$\mathrm{F}$
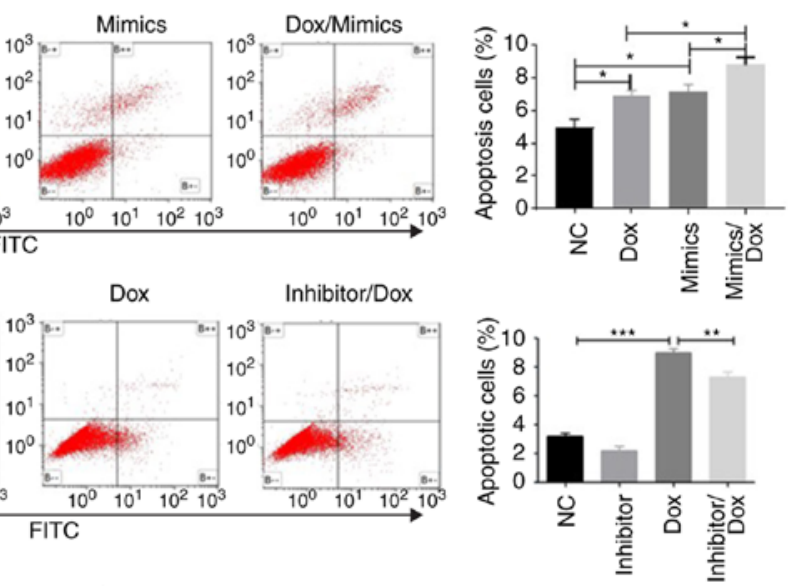

.

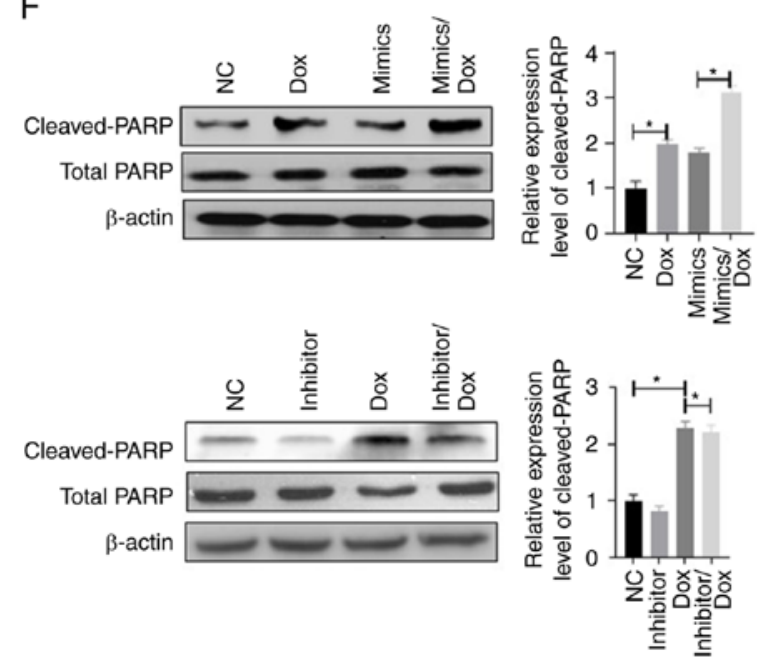

Figure 2. miR-487b-3p negatively regulates osteosarcoma cell migration and chemoresistance. (A) Saos-2 cells were transfected with NC oligonucleotides, miR-487b-3p mimics or miR-487b-3p inhibitor and extracted mRNA were analyzed using reverse transcription-quantitative-PCR, $72 \mathrm{~h}$ following transfection. miR-487b-3p mimics and inhibitors significantly increased and decreased the mRNA expression level of miR-487b-3p in Saos-2 cells, respectively. miR-487b-3p negatively regulates Saos-2 (B) cell migration and (C) inhibits epithelial-mesenchymal transition following transfection with NC, miR-487-3p mimics or inhibitor. (D) miR-487b-3p enhanced the sensitivity of Saos-2 cells to Dox treatment, following transfection with the aforementioned oligonucleotides, treated with or without $0.2 \mu \mathrm{M}$ Dox for $48 \mathrm{~h}$. Cell viability was measured using MTT assay. (E) The overexpression of miR-487b-3p increased Dox-induced apoptosis in Saos-2 cells, while the inhibition of miR-487b-3p inhibited Dox-induced apoptosis in Saos-2 cells, following transfection with the aforementioned oligonucleotides and subsequently treated with DMSO or $0.2 \mu \mathrm{M}$ Dox for $24 \mathrm{~h}$. (F) miR-487b-3p stimulates Dox-induced expression of cleaved- and total-PARP in Saos-2 cells, following transfection with the aforementioned oligonucleotides, and treated with $0.2 \mu \mathrm{M} \mathrm{Dox}$ for $48 \mathrm{~h}$. "P $<0.05$; ${ }^{* *} \mathrm{P}<0.01 ;{ }^{* * *} \mathrm{P}<0.001$. NC, negative control; miR, microRNA; mimics, miR-487b-3p mimics; inhibitor, miR-487b-3p inhibitor; Dox, doxorubicin. 


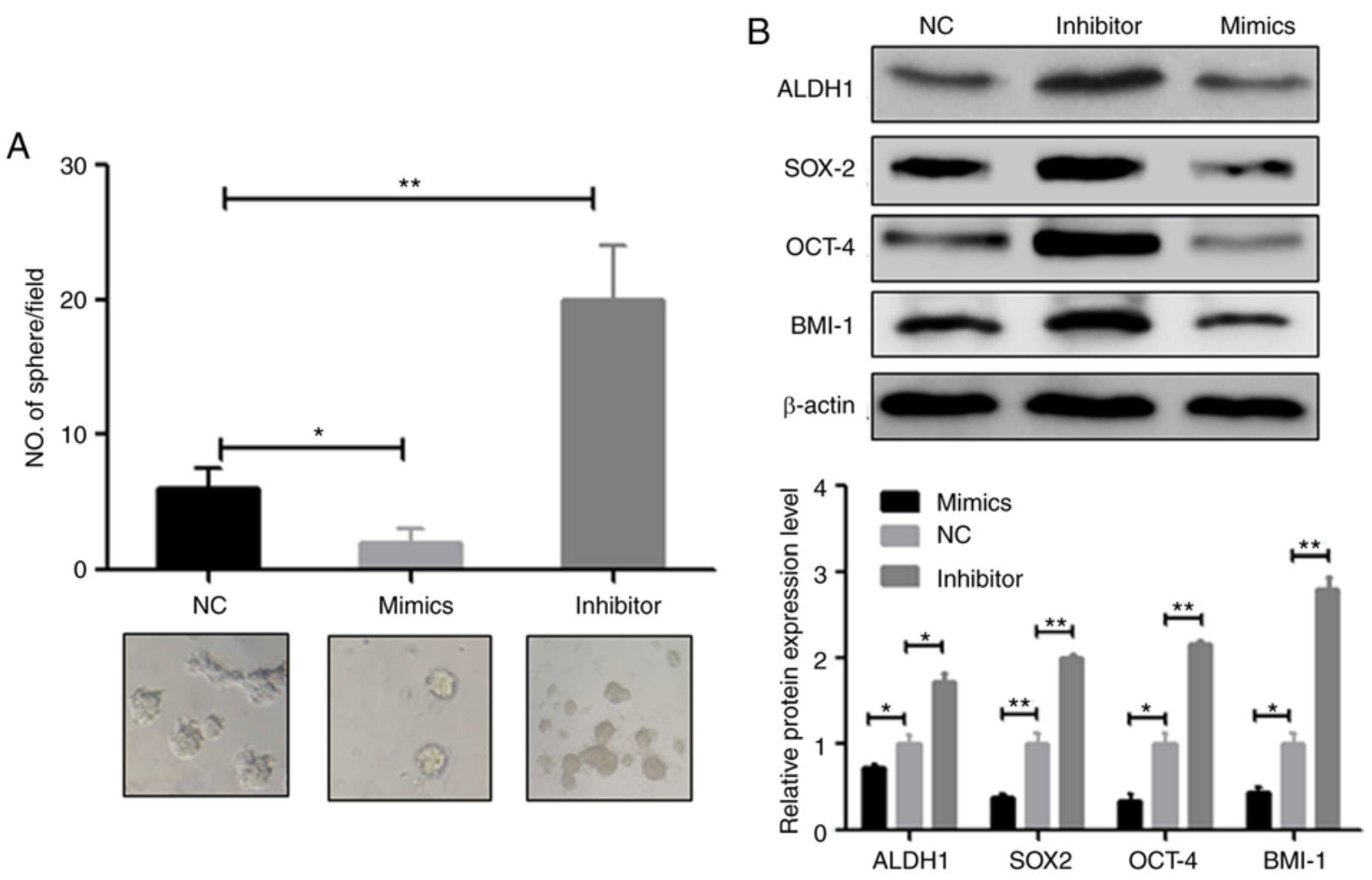

Figure 3. miR-487b-3p negatively regulates osteosarcoma stemness. Saos-2 cells were transfected with either NC oligonucleotides, mimic or inhibitor and were subjected to (A) sphere assay formation or (B) western blot analysis after $24 \mathrm{~h}$ of transfection, where the inhibition and overexpression of miR-487b-3p stimulated and inhibited osteosphere formation in Saos-2 cells, respectively, and miR-487b-3p negatively regulated the protein expression levels of the CSC markers, respectively. ${ }^{*} \mathrm{P}<0.05 ;{ }^{* *} \mathrm{P}<0.01$. NC, negative control; mimics, miR-487b-3p mimics; inhibitor, miR-487b-3p inhibitor.

\section{A}

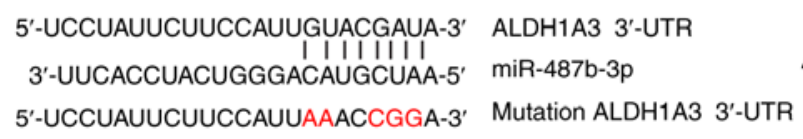

B

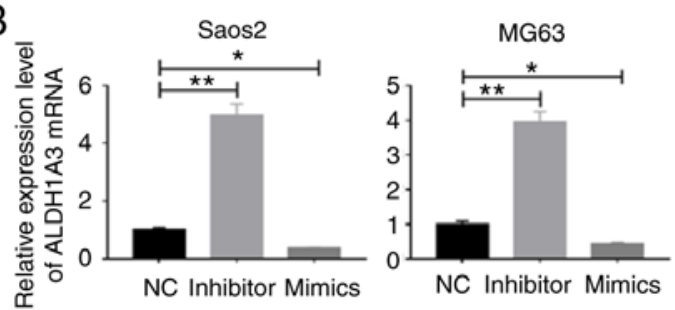

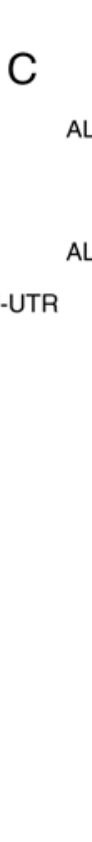

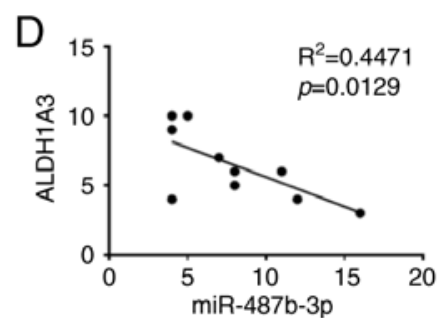

E

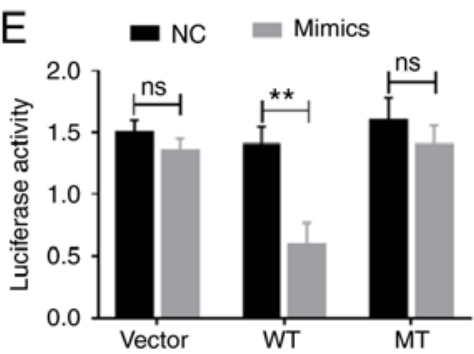

Figure 4. miR-487b-3p targets ALDH1A3. (A) The miR-487b-3p seed sequence is complementary to the 3'UTR of ALDH1A3. Saos-2 and MG63 cells were transfected with either NC, mimic or inhibitor nucleotides and miR-487b-3p inhibited (B) mRNA and (C) protein expression levels of ALDH1A3, 72 h following transfection and were measured using reverse transcription-quantitative PCR and western blot analysis, respectively. (D) The expression levels of ALDH1A3 and miR-487-3p revealed a negative correlation in patients with OS. Tumor samples were obtained from 10 patients with OS. (E) Activity of the luciferase gene linked to the 3'UTR of ALDH1A3. The luciferase reporter plasmids of WT or MT 3'UTR sequences of ALDH1A3 were transfected into 293T cells with or without the mimic. ${ }^{*} \mathrm{P}<0.05$. ${ }^{* *} \mathrm{P}<0.01$. NC, negative control oligonucleotides; mimic, miR-487b-3p mimic; inhibitor, miR-487b-3p inhibitor; ns, no significance; WT, wild-type; MT, mutated; miR, microRNA; ALDH1A3, aldehyde dehydrogenase 1 family member A3. 

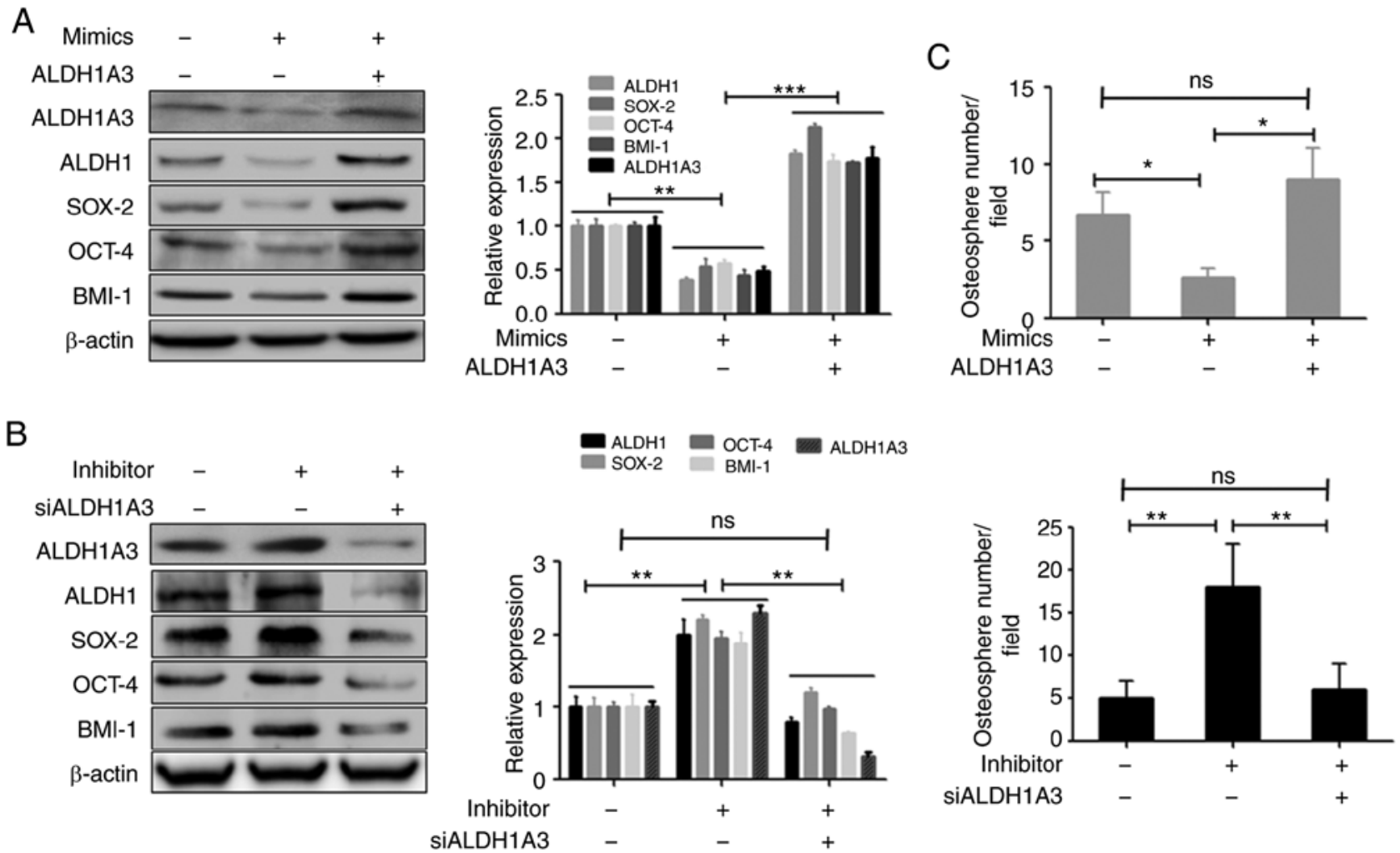

Figure 5. ALDH1A3 causes miR-487b-3p-induced effects on OS stemness. (A) The overexpression of ALDH1A3 increased miR-487b-3p-induced inhibition and (B) The silencing of ALDH1A3 blocked the miR-487b-3p inhibition-induced upregulation of the protein expression levels of CSC markers. Saos-2 cells were transfected with mimic and/or ALDH1A3 expression plasmids or with miR-487b-3p inhibitor and/or ALDH1A3 siRNA, respectively and analyzed using western blot analysis (C) miR-487b-3p regulated OS sphere formation due to ALDH1A3. Saos-2 cells were transfected with the aforementioned nucleotides and plasmids and then subjected to sphere formation assays. ${ }^{*} \mathrm{P}<0.05 ;{ }^{* *} \mathrm{P}<0.01 ;{ }^{* * *} \mathrm{P}<0.001$. NC, negative control; mimic, miR-487b-3p mimic; inhibitor, miR-487b-3p inhibitor; si, short inhibiting; OS, osteosarcoma; ALDH1A3, aldehyde dehydrogenase 1 family member A3; ns, non-significant; +, positive, -, negative; CSC, cancer stem cell.

with miR-487b-3p mimics and subsequently s.c. injected into nude mice to investigate the effect of miR487b-3p on tumorigenesis (Fig. 6A). As expected, treatment with miR-487b-3p blocked CSC-induced tumor formation in nude mice (Fig. 6B). Subsequently, to investigate the effects of miR-487b-3p on chemosensitivity, a xenograft model was generated using ALDH $1^{\text {high }}$ Saos- 2 cells. These mice received three cycles (days 29, 32 and 36) of intraperitoneal injection of Dox, miR-487b-3p or both. The results revealed that Dox treatment inhibited tumor growth, but regrowth of the disease occurred after 40 days. However, no tumor regrowth occurred in the combination treatment group of Dox and miR-487b-3p. (Fig. 6C). In addition, the CSCs in the tumor tissues were investigated, and data revealed that the groups treated with miR-487b-3p had a significantly reduced CSC population compared with that in the groups that were not treated with miR-487b-3p (Fig. 6D).

\section{Discussion}

The occurrence of chemoresistance and metastasis indicates poor prognosis in patients with OS $(3,4)$. Previous studies have revealed that the expression level of these miRNAs (miR-495, miR-329, miR-487b, miR-410, and miR-656) are associated with the progression of OS $(13,14)$. Previous studies have reported that miR-487b-3p suppressed osteoblastic differentiation and the tumorigenicity of colon cancer cells in vivo $(21,22)$. In the present study, an insight into the biological effects of miR-487b-3p in OS metastasis and chemoresistance has been provided. It was found that decreased expression of miR-487b-3p was significantly associated with poor clinical outcomes in patients with OS. In addition, the in vitro results revealed that the inhibition of miR-487b-3p promoted OS cell migration and chemoresistance. The findings from the present study suggests that miR-487b-3p functions as a cancer suppressor and that may be a novel strategy for the treatment of OS metastasis and chemoresistance by the restoration of miR-487b-3p.

In the present study, the anti-OS mechanism of miR-487b-3p was clarified. Accumulated evidence has shown that increased cancer stemness can stimulate cancer metastasis and induce chemoresistance (23-25). The results from the present study showed that the inhibition of miR-487b-3p stimulated OS stemness. Notably, the in vitro and in vivo experiments revealed that the overexpression of miR-487b-3p significantly suppressed OS stemness and number of CSCs in tumor tissues, suggesting that miR-487b-3p plays an anticancer role partially due to the inhibition of CSCs in OS. In addition, the CSC regulation mechanism of miR-487b-3p in OS was investigated, in which a series of in vitro experiments were used to confirm that ALDH1A3 is a target gene of miR-487b-3p in OS. Previous studies have shown that ALDH1A3 is one of the important members of the aldehyde dehydrogenase family, which is a CSC marker and plays an 
A
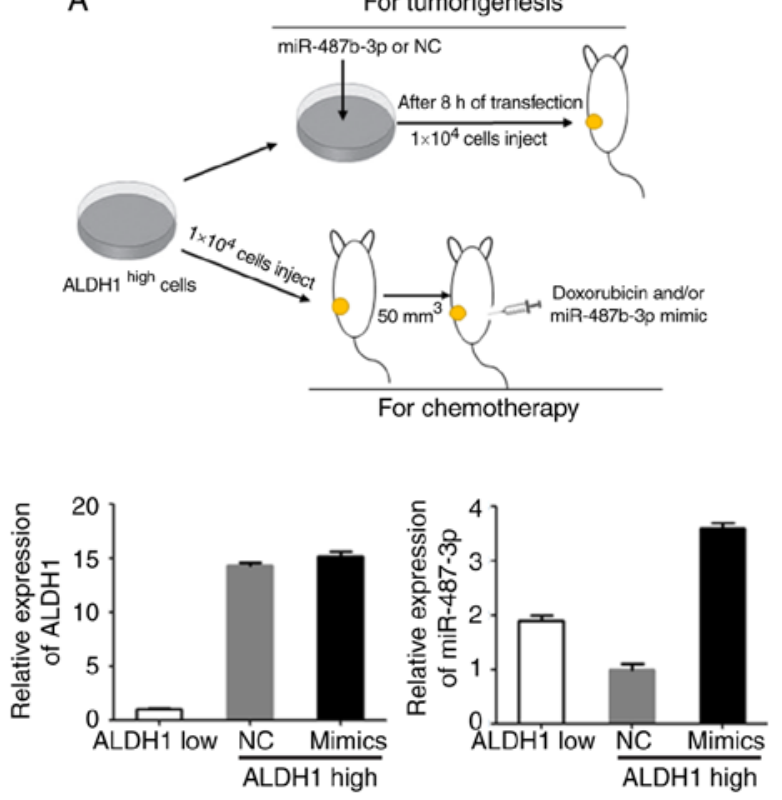

B

\begin{tabular}{lr}
\hline \multicolumn{2}{l}{ Tumor incidence in nude mice } \\
\hline Untreated & $9 / 9$ \\
NC & $8 / 9$ \\
Mimics & $2 / 9$ \\
\hline
\end{tabular}

C
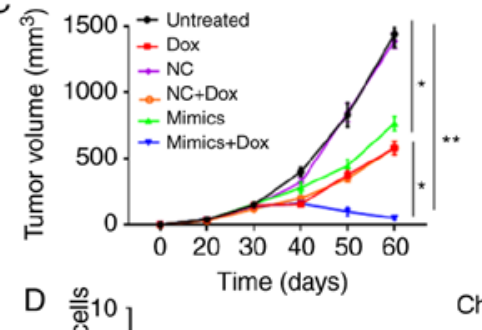

D

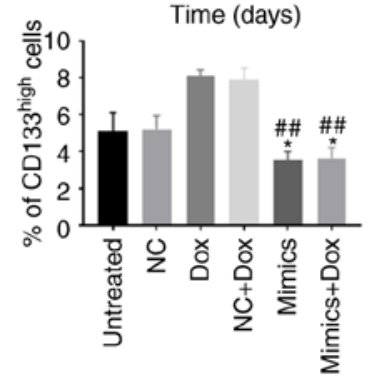

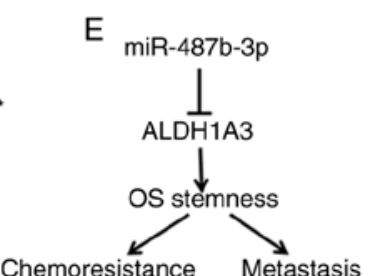

Chemoresistance Metastasis

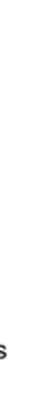

.

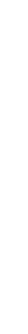

Figure 6. miR-487b-3p overexpression in combination with chemotherapy synergistically inhibits cancer stem cells and prevents tumor relapse. (A) Experimental design, and expression levels of ALDH1 and miR-487b-3p in Saos-2 cells following transfection with mimics. Genes expression was measured after $8 \mathrm{~h}$ of miR-487b-3p transfection. (B) Tumor incidence in nude mice injected with cancer stem cells from Saos-2 cells ( $\mathrm{n}=9 / \mathrm{group}$ ). (C) Tumor volume in xenografts treated with Dox, miR-382 or both on days 29,32 and 36 . ALDH1 ${ }^{\text {high }}$ cells were separated from Saos-2 cells and injected subcutaneously into nude mice ( $\mathrm{n}=9 /$ group). (D) The proportion of CD133 high cells derived from xenograft model tumors. Tumors were collected from xenograft mice ( $\mathrm{n}=3 / \mathrm{group}$ ) 36 days after cell injection. (E) Graphic representation of the mechanism of action of miR-487b-3p to inhibit osteosarcoma chemoresistance and metastasis by targeting ALDH1A3. "P<0.01 compared with untreated group; ${ }^{* *} \mathrm{P}<0.01$ compared with untreated group; ${ }^{\# \#} \mathrm{P}<0.01$ compared with Dox treatment group. NC, negative control; mimic, miR-487b-3p mimic; inhibitor, miR-487b-3p inhibitor; Dox, doxorubicin.

important role in CSC regulation, which can affect the biological characteristics of CSCs through the regulation of other stem cell genes, such as CD133 and OCT4 $(26,27)$. The data in the present study revealed that the ALDH1A3 expression level was increased or decreased in OS cells by the inhibition or increased expression of miR-487b-3p, respectively. In addition, a luciferase reporter assay revealed that miR-487b-3p directly targets the 3'UTR of ALDH1A3. Furthermore, clinical data revealed that the expression levels of miR-487b-3p and ALDH1A3 were inversely correlated in specimens from patient with OS. In additional, the restoration of ALDH1A3 blocked the miR-487b-3p overexpression-induced inhibition of CSCs. Taken together, these data suggest that miR-487b-3p inhibits OS metastasis and chemoresistance through the inhibition of CSCs by targeting ALDH1A3.

In summary, a role for miR-487b-3p in OS chemoresistance and metastasis has been established using a combination of clinical and experimental studies. The overexpression of miR-487b-3p enhances the sensitivity of OS cells to chemotherapy and inhibits OS metastasis through suppressing OS stemness by targeting ALDH1A3 (Fig. 6E). The findings from the present study may also aid in the development of potential therapeutics for the treatment of OS metastasis and chemoresistance.

\section{Acknowledgements}

Not applicable.

\section{Funding}

This study was supported by the National Natural Science Foundation of China (grant nos. 81760393 and 81401790).

\section{Availability of data and materials}

The datasets used and/or analyzed during the current study are available from the corresponding author on reasonable request.

\section{Author's contributions}

MC and MD made substantial contributions to conception and designed the study. ZZG and PGD analyzed and interpreted the data. MC and MD drafted the manuscript and revised it critically for important intellectual content; All authors read and approved the final manuscript.

\section{Ethics approval and consent to participate}

Ethics approval was granted by the Research Ethics Board of the First Affiliated Hospital of Nanchang University (approval no. 2019042). Written informed consent was provided by all the participants.

\section{Patient consent for publication}

Not applicable. 


\section{Competing interests}

The authors declare that they have no competing interests.

\section{References}

1. Messerschmitt PJ, Garcia RM, Abdul-Karim FW, Greenfield EM and Getty PJ: Osteosarcoma. J Am Acad Orthop Surg 17: 515-527, 2009.

2. Meyers PA, Schwartz CL, Krailo M, Kleinerman ES, Betcher D, Bernstein ML, Conrad E, Ferguson W, Gebhardt M, Goorin AM, et al: Osteosarcoma: A randomized, prospective trial of the addition of ifosfamide and/or muramyl tripeptide to cisplatin, doxorubicin, and high-dose methotrexate. J Clin Oncol 23: 2004-2011, 2005.

3. Huang J, Ni J, Liu K, Yu Y, Xie M, Kang R, Vernon P, Cao L and Tang D: HMGB1 promotes drug resistance in osteosarcoma. Cancer Res 72: 230-238, 2012.

4. Marina N, Gebhardt M, Teot L and Gorlick R: Biology and therapeutic advances for pediatric osteosarcoma. Oncologist 9 : 422-441, 2004

5. Xu M, Jin H, Xu CX, Sun B, Song ZG, Bi WZ and Wang Y: miR-382 inhibits osteosarcoma metastasis and relapse by targeting Y box-binding protein 1. Mol Ther 23: 89-98, 2015.

6. Liu C and Tang DG: MicroRNA regulation of cancer stem cells Cancer Res 71: 5950-5954, 2011.

7. MacDonagh L, Gray SG, Breen E, Cuffe S, Finn SP, O'Byrne KJ and Barr MP: BBI608 inhibits cancer stemness and reverses cisplatin resistance in NSCLC. Cancer Lett 428: 117-126, 2018.

8. Iliopoulos D, Lindahl-Allen M, Polytarchou C, Hirsch HA Tsichlis PN and Struhl K: Loss of miR-200 inhibition of Suz12 leads to polycomb-mediated repression required for the formation and maintenance of cancer stem cells. Mol Cell 39: 761-772, 2010.

9. Bartel DP: MicroRNAs: Genomics, biogenesis, mechanism, and function. Cell 116: 281-297, 2004.

10. Song SJ, Poliseno L, Song MS, Ala U, Webster K, Ng C, Beringer $\mathrm{G}$, Brikbak NJ, Yuan X, Cantley LC, et al: MicroRNA-antagonism regulates breast cancer stemness and metastasis via TET-familydependent chromatin remodeling. Cell 154: 311-324, 2013.

11. Ueda T, Volinia S, Okumura H, Shimizu M, Taccioli C, Rossi S, Alder H, Liu CG, Oue N, Yasui W, et al: Relation between microRNA expression and progression and prognosis of gastric cancer: A microRNA expression analysis. Lancet Oncol 11: 136-146, 2010.

12. Jin H, Luo S, Wang Y, Liu C, Piao Z, Xu M, Guan W, Li Q, Zou H, Tan QY, et al: miR-135b stimulates osteosarcoma recurrence and lung metastasis via notch and Wnt $/ \beta$-catenin signaling. Mol Ther Nucleic Acids 8: 111-122, 2017.

13. Thayanithy V, Sarver AL, Kartha RV, Li L, Angstadt AY, Breen M, Steer CJ, Modiano JF and Subramanian S: Perturbation of $14 \mathrm{q} 32$ miRNAs-cMYC gene network in osteosarcoma. Bone 50: 171-181, 2012.
14. Hill KE, Kelly AD, Kuijjer ML, Barry W, Rattani A, Garbutt CC, Kissick H, Janeway K, Perez-Atayde A, Goldsmith J, et al: An imprinted non-coding genomic cluster at $14 \mathrm{q} 32$ defines clinically relevant molecular subtypes in osteosarcoma across multiple independent datasets. J Hematol Oncol 10: 107, 2017.

15. Marcato P, Dean CA, Pan D, Araslanova R, Gillis M, Joshi M, Helyer L, Pan L, Leidal A, Gujar S, et al: Aldehyde dehydrogenase activity of breast cancer stem cells is primarily due to isoform ALDH1A3 and its expression is predictive of metastasis. Stem Cells 29: 32-45, 2011.

16. Sullivan KE, Rojas K, Cerione RA, Nakano I and Wilson KF: The stem cell/cancer stem cell marker ALDH1A3 regulates the expression of the survival factor tissue transglutaminase, in mesenchymal glioma stem cells. Oncotarget 8: 22325-22343, 2017.

17. Duan JJ, Cai J, Guo YF, Bian XW and Yu SC: ALDH1A3, a metabolic target for cancer diagnosis and therapy. Int J Cancer 139: 965-975, 2016.

18. Biermann JS, Chow W, Reed DR, Lucas D, Adkins DR, Agulnik M, Benjamin RS, Brigman B, Budd GT, Curry WT, et al: NCCN Guidelines Insights: Bone Cancer, Version 2.2017. J Natl Compr Canc Netw 15: 155-167, 2017.

19. Steffner RJ and Jang ES: Staging of bone and soft-tissue sarcomas. J Am Acad Orthop Surg 26: e269-e278, 2018.

20. Livak KJ and Schmittgen TD: Analysis of relative gene expression data using real-time quantitative PCR and the 2(-Delta Delta C(T)) method. Methods 25: 402-408, 2001.

21. Yi H, Geng L, Black A, Talmon G, Berim L and Wang J: The miR-487b-3p/GRM3/TGF $\beta$ signaling axis is an important regulator of colon cancer tumorigenesis. Oncogene 36: 3477-3489, 2017.

22. John AA, Prakash R and Singh D: miR-487b-3p impairs osteoblastogenesis by targeting Notch-regulated ankyrin-repeat protein (Nrarp). J Endocrinol 241: 249-263, 2019.

23. Brabletz T, Jung A, Spaderna S, Hlubek F and Kirchner T: Opinion: Migrating cancer stem cells-an integrated concept of malignant tumour progression. Nat Rev Cancer 5: 744-749, 2005.

24. Li F, Tiede B, Massagué J and Kang Y: Beyond tumorigenesis: Cancer stem cells in metastasis. Cell Res 17: 3-14, 2007.

25. He H, Ni J and Huang J: Molecular mechanisms of chemoresistance in osteosarcoma (Review). Oncol Lett 7: 1352-1362, 2014.

26. Sullivan KE, Cerione RA and Wilson KF: ALDH1A3 in CSCs. Aging (Albany NY) 9: 1351-1352, 2017.

27. Xu X, Chai S, Wang P, Zhang C, Yang Y, Yang Y and Wang K: Aldehyde dehydrogenases and cancer stem cells. Cancer Lett 369: 50-57, 2015. 